\title{
Editorial
}

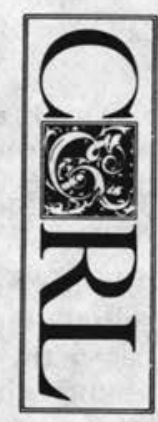

\section{Worlds of Reference}

"It's natural!" I proclaimed quickly. Just as quickly I regretted my words. Ben Amata, the government documents librarian, smiled. After I clarified my meaning in order to remove the strong pejorative bias, we continued our discussion about the pros and cons of classifying periodicals. At Sacramento periodicals are shelved in title order, but it has been suggested that we classify and order them by call number prior to the move into a major new addition.

My "natural" comment was elicited because students are familiar with alphabetical arrangements for organizing things. It is ingrained in them from childhood. The cubbyhole of my daughter, Jennifer, comes before Joshua's and after Hillary's at the Little Learners Daycare. Filing by title lets users go directly from periodical index to shelf and avoids the need for users to understand or jot down an artificial and thus alien call number.

My regret came because if what is natural is right, then what is unnatural must be wrong. However, people who regularly use this argumentative technique will soon lack an audience. I wanted dialogue, not silence. I did not want to impose an alienating structure on the problem any more than I want to impose an alienating organizing structure on our users.

Most of us have heard the advice that we should not organize our online catalogs like our card catalogs. We should take advantage of the new opportunities inherent in the new technologies to create new or better organizing principles. I always felt that this advice was sound, but I never knew why. I also wondered why I had only an intuition or a sense of the correctness of this advice but not a clearer, more objective knowledge.

Several days after my conversation with Ben, while reading Tom McArthur's Worlds of Reference: Lexicography, Learning and Language from the Clay Tablet to the Computer (London: Cambridge Univ. Pr., 1986), I learned why. Or more correctly, I relearned why.

Through the process of acculturation our minds become structured so that "things" are viewed from a certain perspective or set of perspectives. The problems that we face are unconsciously placed within this broader mental structure. Some would say that this process is necessary so that we can conclude quickly and act quickly. However, in some cases this mental structure may be inappropriate for the problem at hand.

Perhaps some of you know someone whose mind seems to operate as if it contained the knowledge of only one book, memorized once, and closed forever. The way this person thinks about things is obvious to us after only a few exchanges. We think of him or her as inflexible or rigid. For all of us, however, mental structures serve to delimit or cut us off from seeing some things in certain ways.

I tended to think of alphabetization as a natural ordering process until I read McArthur's words: "The catalogues have been compiled by a nineteenth century man of science and carry the inevitable mark of their time, but all in all they serve to remind us that whatever does happen in our minds when we order and retrieve 'words' and 'ideas,' it is not done alphabetically".

I don't know how to acquire a more responsive, better set of mental structures for organizing things, whether in life or libraries. I will try because the new perspectives may en- 
able me to understand or to create a viable context for understanding what computers and other advanced technologies are doing to our world. Like a climber I need something to hold onto if I hope to climb higher. Maybe something simple will appear, something just as simple as the climber's piton.

According to McArthur, the consequences of the arrival of printing "were so vast that they have virtually wiped out our ability to understand what life was like in the scribal cultures that preceded printing". If the computer revolution parallels or exceeds the impact of the printing press on civilization we are going to have to challenge all our mental resources just to pick up the occasional glimmers of understanding that may slip through the dense fog that hides the future from us.

CHARLES MARTELL

\section{IN FORTHCOMING ISSUES OF COLLEGE \& RESEARCH LIBRARIES}

Special Collections: Strategies for Support in an Era of Limited Resources by Rebecca Martin

Library Literature in Mainland China: A Content Analysis by Douglas Cooper

Learning the Library: Taxonomy of Skills and Errors by Leon Jakobovits and Diane Nahl-Jakobovits

The Academic Librarian and Faculty Status in the 1980s

by Kee DeBoer and Wendy Culotta

Cooperation, Collection Management, and Scientific Journals by Elizabeth P. Roberts 